Opinion

\title{
Painless, reproducible, reversible floor of mouth herniation: what's in there?
}

\section{Opinion}

\section{Description}

An 18year old female presented with a left submandibular swelling (Figure 1) which was diagnosed to be a plunging ranula and surgery was recommended. The patient moved abroad and as the neck lump disappeared spontaneously no active intervention was ever sought. Ten years later, she represented with a right submandibular swelling which fluctuated in size over the preceding 4months but had failed to disappear completely. The clinical examination revealed a soft, cystic swelling in the right submandibular region (Figure 2). The clinical diagnosis of right plunging ranula was made with no intra-oral component (Figure 3 ). The Ultrasound scan revealed a complex cyst (measuring $6 \times 4 \times 2 \mathrm{~cm}$ ) sitting in front of a normal right submandibular gland. Two weeks before her planned surgery, she developed acute infection involving her right floor of mouth with significant neck swelling. She responded well to intravenous antibiotics and was discharged home the following day on oral antibiotics.

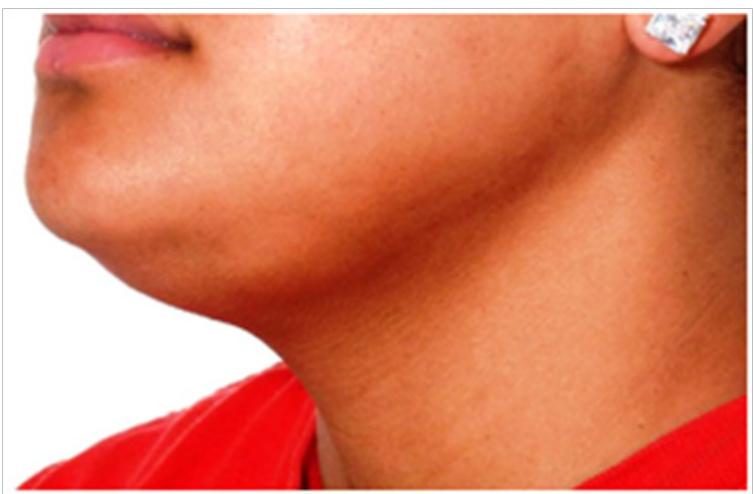

Figure I Left submandibular swelling-Left plunging ranula.

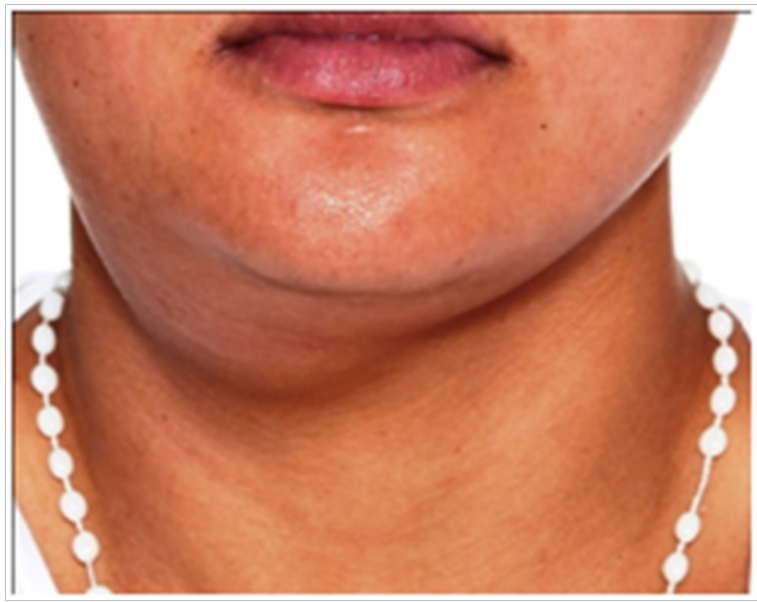

Figure 2 Right submandibular swelling-Right plunging ranula.
Volume 4 Issue 6 - 2016

\section{Zahoor Ahmad, Shakeel M, Nair S}

Department of Otolaryngology-Head and Neck Surgery, Super Clinic, New Zealand

\section{Correspondence: Zahoor Ahmad, Consultant}

Otolaryngologist, Department of Otolaryngology- Head and Neck Surgery, Manukau Super Clinic, PO Box 98743, South Auckland Mail Center, Auckland 224I, New Zealand,Tel +64212137576, Email zaheer.ahmad@middlemore.co.nz

Received: August 28, 2016 | Published: October 14, 2016

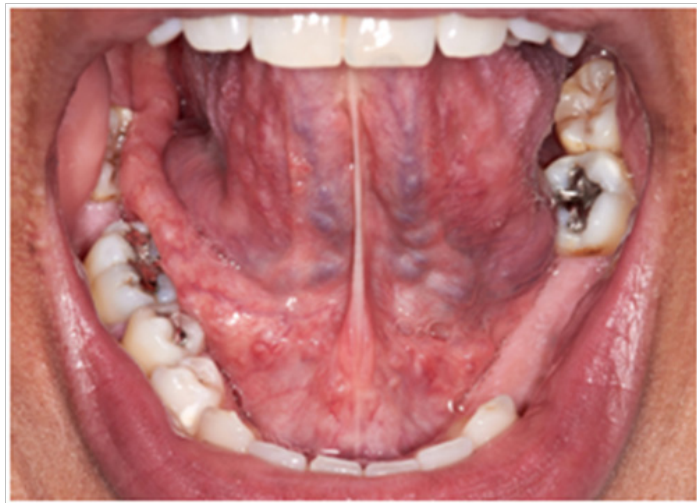

Figure 3 Normal floor of mouth.

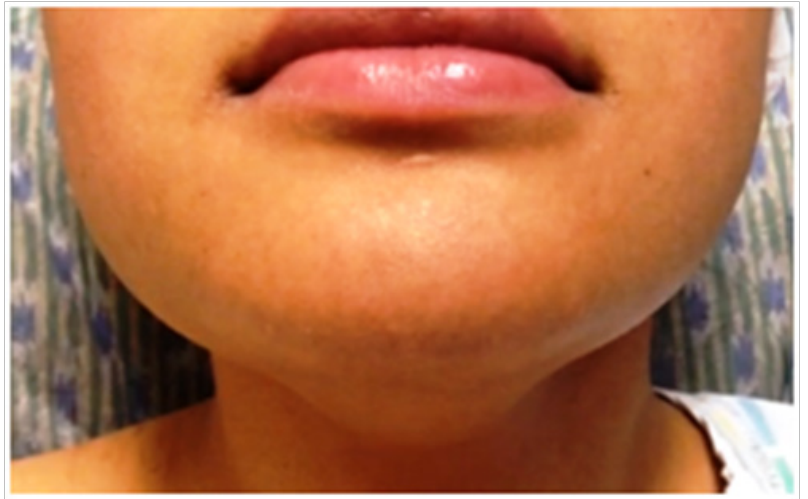

Figure 4 No visible submandibular regions swelling.

On the day of surgery, no residual swelling could be seen in her neck at rest (Figure 4). However, the patient volunteered to reproduce 
her neck swelling (Video 1). To our surprise, there were bilateral submandibular swellings which became apparent on modified valsalva manoeuvre and spontaneously reduced without causing any discomfort to the patient. The patient underwent transoral excision of the right sublingual salivary gland and drainage of the associated cyst under general anaesthetic. The patient made excellent recovery and was discharged home the same day.

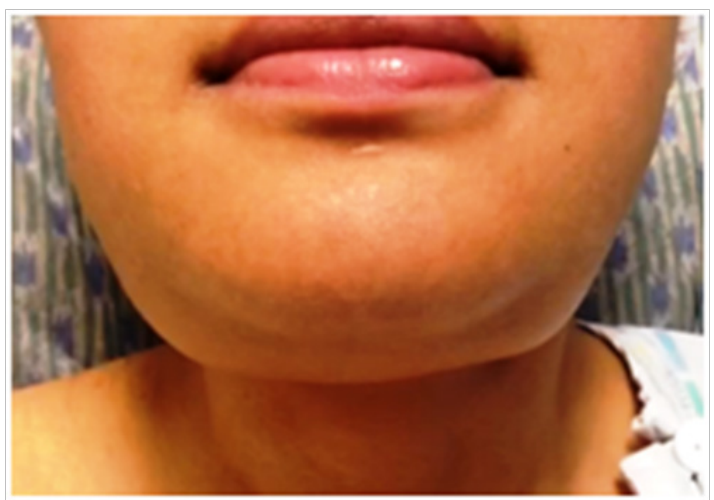

Figure 5 Bilateral submandibular swellings secondary to herniation of the sublingual glands.

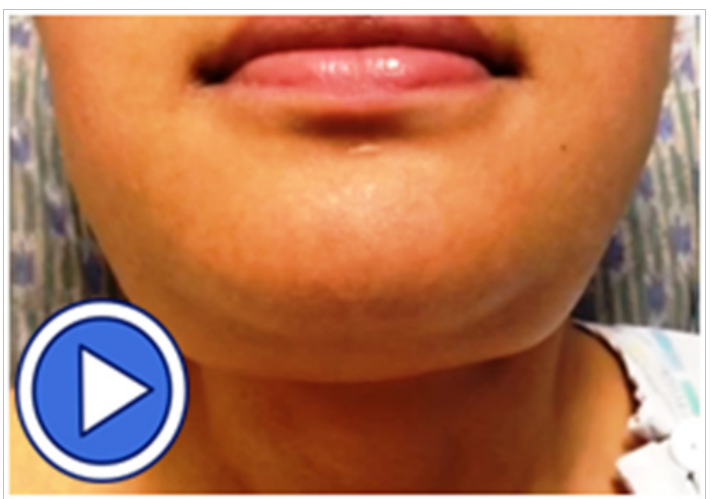

Video: Click here on above video.
Plunging ranula is a pseudocyst formed by extravasation of mucous from the sublingual gland (SLG) into the submandibular space. It is thought to have a congenital aetiology. ${ }^{1}$ The plunging ranula is suspected clinically and confirmed; radiologically by Ultrasound, ${ }^{2}$ CT or MRI scan, and by fine needle aspiration for cytology or biochemical analysis confirming presence of salivary amylase in the aspirate. Based on the published literature, trans-oral excision of the SLG is favoured to treat the plunging ranula. ${ }^{3}$

\section{Learning points}

a. Bilateral plunging ranula is a rare phenomenon but is an important differential diagnosis for submandibular region swellings.

b. Bilateral reversible sublingual gland herniation through the mylohyoid muscles defect without associated ranula does not always need removal of gland.

c. Transoral excision of the sublingual salivary gland with drainage of the cystic contents is the recommended treatment for plunging ranula.

\section{Acknowledgments}

None.

\section{Conflicts of interest}

Author declares there are no conflicts of interest.

\section{Funding}

None.

\section{References}

1. Sastre J, Quirse S. Occupational allergic disease. 2013.

2. Meredith SK, Taylor DM. Most occupational respiratory disease in the UK. 2011.

3. Report British Thoracic Society of Occupational Medicine.

4. WHO; report of occupational rhinitis. 2011. 